
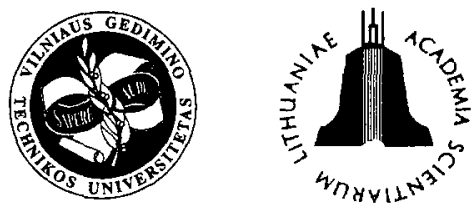

ISSN $1392-3730$

JOURNAL OF CIVIL ENGINEERING AND MANAGEMENT

http:/www.vtu.lt/english/editions

2002, Vol VIII, No 3, 143-152

\title{
OPTIMIZATION OF LAMINATED BENDING PLATES
}

\author{
Dainius Rusakevičius!, Rimantas Belevičius ${ }^{2}$ \\ Dept of Engineering Mechanics, Vilnius Gediminas Technical University, Sauletekio al. 11, \\ LT-2040Vilnius, Lithuania.'E-mail:dairus@fm.vtu.lt'2E-mail:rb@fm.vtu.lt
}

Received 20 June 2001; accepted 15 May 2002

\begin{abstract}
The problems of shape, material, sizing and topology optimization are formulated and solved for thin orthotropic laminated bending plate structures subjected to the in-plane as well as out-of-plane loading. A simple laminated combined finite element that comprises known elements CST for membrane part and DKT for bending part was formulated in the present study. A special numerical-analytical method for structural matrices of the element and analytical sensitivity analysis is used to cope with huge required computer resources for topology optimization. The uniform distribution of deformation energy density over the area of structure is employed as the optimality criterion in all optimization problems. The original algorithms, which may be attributed to the ESO family of algorithms, are suggested for optimization of laminae thicknesses and topology optimization. A number of numerical examples are provided.
\end{abstract}

Keywords: thin laminated bending plate, optimization of shape, material, sizing and topology, finite element method.

\section{Introduction}

The use of laminated structures is receiving even wider attention and use in commercial applications. Due to superior mechanical properties and low weight laminated composite materials are finding a wide range of applications in automotive industry, aviation, etc. Almost all practical composite material structures are orthotropic and thin in the thickness direction because their superior properties permit the use of thin-walled structures. Due to the inherent tailoring of properties of these materials, a number of unique design features can be utilized including such potential aspects as reduced weight, performance, increased service life and reduced system maintenance. Tailoring of a laminated composite structure that efficiently meets the requirements of certain applications can be achieved by varying a large group of parameters, such as thicknesses of structural members, orientations of orthotropy axes in plies, the number and stacking sequence of plies, the shape of structural members, and, finally, the topology of whole (or of certain part of) structure.

The present paper deals with aforementioned optimization problems of laminated orthotropic plate structures. The optimization problems of two-dimensional plane stress or plane strain structures deserved a wide attention of investigators in recent years, therefore we concentrate ourselves on shape, material, and topology optimization of thin bending laminated plate structures subjected to in-plane as well as out-of-plane loads, and composed of an arbitrary number of orthotropic plies.
Only a few scientific publications are available on this subject [1]. The problems of shape, material and topology optimization are formulated in the paper. In the material optimization, the orthotropy orientation angles of plies, plies stacking sequence, and thicknesses of plies can be varied. It is hardly conceivable that the last problem will find practical applications, because it results in step-wise shape cross-section of plate. However, that formulation is used in topology optimization algorithms. In all problems the deformation energy density is used as a criterion function, seeking for its uniform distribution over all structure.

Original algorithms were employed for shape, crosssection and topology optimization. The last algorithm is based on Optimality Criterion (OC) [2] and Evolutionary Structural Optimization (ESO) [3] methods. Numerical examples illustrate the suggested algorithms in action.

\section{Methods of structural optimization}

Structural optimization can be categorized into four basic types (Fig 1):

1) Sizing optimization

2) Material optimization

3) Shape optimization

4) Topology optimization

The differences between optimization categories mainly consist in the definition of design variables, ie parameters that are changed during the optimization process seeking for optimal value of a chosen optimum criterion. 
Sizing optimization. In the sizing optimization, the layout of the structure is prescribed. The structure is characterised by a sequence of dimensions that comprises the design parameters set. Aim of optimization is the combination of dimensions by which the optimum criterion exposes the lowest possible magnitude [4].

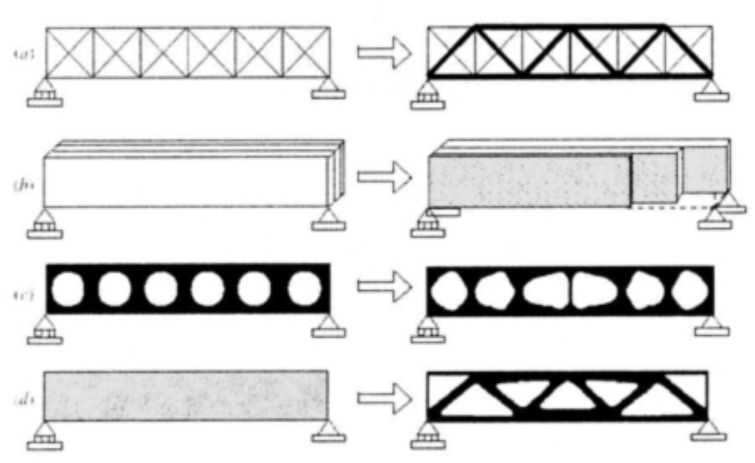

Fig 1. Categories of structural optimization [4]

Material optimization. The material and cross-section layout characteristics are the design parameters for this problem. The orthotropy orientation angles in particular layers, thicknesses of layers, layers' stacking sequence, etc [4] may be chosen as the design parameters for laminated structures.

Shape optimization. With shape optimization the topology of the structure is known and unchanged during optimization process. The object of optimization is to find the best shape that will have the best optimum criterion outcome. Only the parameters on which the shape of structure depends (eg coordinates of contour in simplest case) are chosen as the design parameters. There are several techniques for solving the problem: mathematical programming, evolutionary structural optimization, and simulated biological growth [5-9].

Solutions obtained by sizing, material and shape optimization methods maintain the same topology as the initial design, and may be far from optimal because other competing topologies cannot be explored. Topology optimization exists where the actual form of the structure is unknown in advance, and the fundamental scheme of structure determined by a whole of structural members, joints of members, boundary conditions is sought. For these reasons, topology optimization is becoming an increasingly important tool in engineering design. There are currently three major topology optimization methods, all using strategies that fully discretize an entire design domain with dense finite element mesh, and then degrade stiffness from its maximum to an acceptable level by gradually removing elements or material:

1) Evolutionary Structural Optimization method (ESO) [3] and its variants. Starting from a dense initial mesh, particular elements are removed from the mesh through a lengthy solution procedure. A fundamental drawback of the method is the strong dependence of the solution on the initial mesh from which it is evolved. In the sequence of elements removal the situation may occur, when the removed element should be reintroduced into the structure. The capability to reinstitute elements has been added to the Bidirectional Evolutionary Structural Optimization (BESO) [10-12], however this addition is still restricted to previous element positions.

2) Homogenization methods [13-15]. These methods introduce composites with perforated microstructures of continuously varying density and orientation (controlled by two or more local parameters) as admissible materials for the structural design. For each element these parameters become the design variables. The mathematical programming methods are used to determine the optimal material distribution. The resulting highly perforated structures, which have various sizes of microholes in various orientations or various densities, have to be geometrically and structurally interpreted to give a meaningful layout.

3) Genetic Algorithms (GA) [16-17] are based on the theory of natural selection. The structural topology optimization can be achieved by successive ranking of populations of elements and elimination of the weakest. From the selected group more populations are generated and again ranked. Also, at each elimination some poorly ranked elements are "mutated" with highly ranked elements to avoid "in-breeding". So the optimum emerges.

In the present work optimization problems of all aforementioned categories are formulated and solved for thin laminated orthotropic bending plates. The finite element, combined from elements DKT and CST [18] and comprising all essential membrane-bending interactions, is formulated and used for the solutions.

\section{Integrated element DKT and CST}

Triangular integrated element DKT and CST possess 3 nodes with 6 degrees of freedom each: 3 displacements, 2 rotations about in-plane axes and 1 fictitious rotation about out-of-plane axis. Finite element is formulated analytically in closed form.

\subsection{Structural matrices of element}

Stiffness matrix consists of membrane, bending and membrane-bending interaction parts:

$$
\left[K_{i j}\right]=\left[\begin{array}{ll}
{\left[K_{i j}^{p l}\right]} & {\left[K_{i j}^{p l b}\right]} \\
\operatorname{sym} . & {\left[K_{i j}^{b}\right]}
\end{array}\right],
$$

\section{$i, j=1,2,3$.}

Membrane stiffness submatrix for element with arbitrary oriented orthotropic layers is as follows: 
$\left[K^{p l}\right]_{i j}=\frac{1}{4 A}\left[\begin{array}{cc}b_{j}\left(d_{11}^{p l} b_{i}+d_{31}^{p l} c_{i}\right)+ & c_{j}\left(d_{12}^{p l} b_{i}+d_{32}^{p l} c_{i}\right)+ \\ +c_{j}\left(d_{13}^{p l} b_{i}+d_{33}^{p l} c_{i}\right) & +b_{j}\left(d_{13}^{p l} b_{i}+d_{33}^{p l} c_{i}\right) \\ b_{j}\left(d_{21}^{p l} c_{i}+d_{31}^{p l} b_{i}\right)+ & c_{j}\left(d_{22}^{p l} c_{i}+d_{32}^{p l} b_{i}\right)+ \\ +c_{j}\left(d_{23}^{p l} c_{i}+d_{33}^{p l} b_{i}\right) & +b_{j}\left(d_{23}^{p l} c_{i}+d_{33}^{p l} b_{i}\right)\end{array}\right]$

$i, j=1,2,3$,

here $A$ is for element area, $d^{m l}$ is for accumulative elasticity matrix, and $b_{i}, c_{i}$ are geometrical coefficients of element CST, indeed differences of nodal coordinates.

The $i j$-th element of bending stiffness submatrix can be expressed through interpolation functions $N$ of second-order membrane finite element LST [19]:

$$
\begin{aligned}
& K_{i j}^{b}=\frac{1}{4 A^{2}} \sum_{i i=1}^{3} \sum_{j j=1}^{3} \sum_{i i i=1}^{3} \sum_{j j j=1}^{3}\left[\left(X _ { i , i i } b _ { i i i } \left(d_{11}^{b} X_{j, j j} b_{j j j}+\right.\right.\right. \\
& \left.d_{12}^{b} Y_{j, j j} c_{j j j}+d_{13}^{b}\left(X_{j, j j} c_{j j j}+Y_{j, j j} b_{j j j}\right)\right)_{+} \\
& Y_{i, i i} c_{i i i}\left(d_{21}^{b} X_{j, j j} b_{j j j}+d_{22}^{b} Y_{j, j j} c_{j j j}+\right. \\
& \left.d_{23}^{b}\left(X_{j, j j} c_{j j j}+Y_{j, j j} b_{j j j}\right)\right)+ \\
& \left(X_{i, i i} c_{i i i}+Y_{i, i i} b_{i i i}\right)\left(d_{31}^{b} X_{j, j j} b_{j j j}+\right. \\
& \left.\left.d_{32}^{b} Y_{j . j j} c_{j j j}+d_{33}^{b}\left(X_{j, j j} c_{j j j}+Y_{j, j j} b_{j j j}\right)\right)\right) \times \\
& \left.\int_{A}^{\partial N_{i i z} \partial N_{j j z}} \partial L_{i i i} \partial L_{j j j}\right], \\
& i, j=1,2, \ldots, 9,
\end{aligned}
$$

where $[X]$ and $[Y]$ arise after excluding in the interpolation functions of element DKT (formulated in area-coordinates $L$ ) the parts depending on the integral over element volume. Indices $i i z$ and $j j z$ here and below are figured up accordingly to the rules of integer division.

The stiffness elements for membrane-bending interaction are:

$$
\begin{aligned}
& K_{(2 i-1) j}^{p l b}=\frac{1}{4 A^{2}} \sum_{j j=1}^{3} \sum_{j j j=1}^{3}\left[\left(x_{j, j j} b_{j j j}\left(d_{11}^{p l b} b_{i}+d_{31}^{p l b} c_{i}\right)+\right.\right. \\
& Y_{j, j j} c_{j j j}\left(d_{12}^{p l b} b_{i}+d_{32}^{p l b} c_{i}\right)\left(x_{j, j j} c_{j j j}+Y_{j, j j} b_{j j j}\right) \times \\
& \left.\left.\left(d_{13}^{p l b} b_{i}+d_{33}^{p l b} c_{i}\right)\right) \int_{A}^{\partial} \frac{\partial N_{j j z}}{\partial L_{j j j}} d \tau\right], \\
& K_{(2 i) j}^{p l b}=\frac{1}{4 A^{2}} \sum_{j j=1}^{3} \sum_{j j j=1}^{3}\left[\left(X_{j, j j} b_{j j j}\left(d_{21}^{p l b} c_{i}+d_{31}^{p l b} b_{i}\right)+\right.\right. \\
& Y_{j, j j} c_{j j j}\left(d_{22}^{p l b} c_{i}+d_{32}^{p l b} b_{i}\right)+\left(X_{j, j j} c_{j j j}+Y_{j, j j} b_{j j j}\right) \times \\
& \left.\left.\left(d_{23}^{p l b} c_{i}+d_{33}^{p l b} b_{i}\right)\right) \int_{A}^{\partial N_{j j j z}} d \tau\right], \\
& \left.i=1,2,3, \quad j L_{j j j}\right]
\end{aligned}
$$

The integrals in (3) and (4) have to be calculated beforehand by means of computer algebra.

The suggested method [19] of finite element formulation, when all structural matrices are obtained avoiding the procedure of numerical integration over element volume, is considerably more economical in regard of computing resources and therefore is chosen for all optimization problems.

\section{Shape, material and cross-section optimization of laminated bending plate}

\subsection{Sensitivity analysis}

The calculation of the sensitivity of structural responses to changes in design variables is the major computational cost of the optimization process. Therefore the efficient numerical algorithms for all necessary derivatives is the must. The mostly chosen technique is the finite-difference approximation. In the present work another method was used. Analytical expressions were obtained for all necessary derivatives. This allows for high accuracy of calculations, which is especially imperative in shape and cross-section optimization of purely plate bending problems. In this case, when the plate is loaded only by out-of-plane loads, all necessary information for the shape and cross-section changes is given only by membrane-bending interaction stiffness that is significantly lower than other stiffnesses.

The sensitivity analysis yields the influence of design parameters to the optimum criterion. For that the derivatives of finite element stiffness matrix, of element area, of stress vector, and of density of deformation energy were obtained with regard to all design parameters: nodal coordinates of contour, orthotropy angles of laminae, and thickness coefficients of laminae.

The general sensitivity equation for whole structure is:

$$
[K]_{\partial} \frac{\partial \delta}{t_{m}}=\mathbf{P},
$$

$m=1,2, \ldots, n$,

with $t$ for the design parameter, $n$ for the number of design parameters and $\boldsymbol{\delta}$ for the vector of nodal displacements. The so-called pseudo-load vector is determined by:

$$
\mathbf{P}=\frac{\partial \mathbf{P}}{\partial t_{m}}-\frac{\partial[K]}{\partial t_{m}} \mathbf{\delta} .
$$

The (5) and (6) yield the displacement derivatives that are present in all remaining expressions of sensitivity analysis.

\subsection{Finite element derivatives}

Derivatives of membrane part of stiffness matrix.

In-plane behaviour of plate is modelled by wellknown Constant Strain Triangle (CST) element. The stiffness coefficients of element are indeed the differences of nodal coordinates, therefore the derivatives can be easily obtained and are not shown here. The same holds to the element area. 
Derivatives of bending part of stiffness matrix.

Out-of-plane behaviour of plate is modelled by Discrete Kirchhoff theory (DKT) element. General expressions of derivatives with regard to coordinates $x$ and $y$ of design nodes are as follows:

$$
K_{i j, t_{m}}^{b}=\frac{1}{2 A}\left(K_{i j}^{* b},_{m}-2 K_{i j}^{b} A_{, l_{m}}\right) .
$$

Hence, the derivative with regard to coordinate $x$ is:

$$
\begin{aligned}
& K_{i j}^{* b} x_{m}=\sum_{i i=1}^{3} \sum_{j j=1}^{3} \sum_{i i i=1}^{3} \sum_{j j j=1}^{3}\left[\left(c _ { i i i . . x _ { m } } \left(d_{22} c_{j j j} Y_{i, i i} Y_{j, j j}+\right.\right.\right. \\
& d_{12} b_{j j j} Y_{i, i i} X_{j, j j}+d_{33} c_{j j j} X_{i, i i} X_{j . j j}+d_{33} b_{j j j} X_{i, i i} Y_{j, j j}+ \\
& c_{j j j_{, r_{m}}}\left(d_{22} c_{i i i} Y_{i, i i} Y_{j, j j}+d_{12} b_{i i i} X_{i, i i} Y_{j, j j}+\right. \\
& \left.d_{33} c_{i i i} X_{i, i i} X_{j, j j}+d_{3,3} b_{i i i} Y_{i, i i} X_{j, j j}\right)+ \\
& X_{i, i i_{, r_{m}}}\left(d_{11} b_{i i i} b_{j j j} X_{j, j j}+d_{12} b_{i i i} c_{j j j} Y_{j, j j}+\right. \\
& \left.d_{33} c_{i i i} c_{j j j} X_{j, j j}+d_{33} c_{i i i} b_{j j j} Y_{j, j j}\right)+ \\
& X_{j, j j_{, x_{m}}}\left(d_{11} b_{i i i} b_{j j j} X_{i, i i}+d_{12} c_{i i i} b_{j j j} Y_{i, i i}+\right. \\
& \left.d_{33} c_{i i i} c_{j j j} X_{i, i i}+d_{33} b_{i i i} c_{j j j} Y_{i, i i}\right)+ \\
& Y_{i, i i}, x_{i n}\left(d_{22} c_{i i i} c_{j j j} Y_{j, j j}+d_{12} c_{i i i} b_{j j j} X_{j, j j}+\right. \\
& \left.d_{33} b_{i i i} c_{j j j} X_{j, j j}+d_{33} b_{i i i} b_{i j j} Y_{j, j j}\right)+ \\
& Y_{j, j j_{. x_{m}}}\left(d_{22} c_{i i i} c_{j j j} Y_{i, i i}+d_{12} b_{i i i} c_{j j j} X_{i, i i}+\right. \\
& \left.\left.\left.d_{33} c_{i i i} b_{j j j} X_{i, i i}+d_{33} b_{i i i} b_{j j j} Y_{i, i i}\right)\right) \int_{A}^{\partial N_{i i z} \partial N_{j j z}} d \tau\right] \text {. }
\end{aligned}
$$

$i, j=1,2, \ldots, 9$.

The adequate derivatives with regard to $\mathrm{y}$ may be obtained exchanging in (8) derivatives $[X]_{x}$ by $[Y]_{, y}$.

Derivatives of membrane-bending interaction stiffnesses.

Analogously, we obtain

$$
\begin{aligned}
& K_{(2 i-1) j_{, x_{m}}^{*}}^{* p l h}=\sum_{j j=1}^{3} \sum_{j j j=1}^{3}\left[\left(c_{i, x_{m}}\left(d_{33} c_{j j j} X_{j, j j}+d_{33} b_{j j j} Y_{j, j j}\right)+\right.\right. \\
& c_{j j j_{, x_{m}}}\left(d_{12} b_{i} Y_{j, j j}+d_{33} c_{i} X_{j, j j}\right)+ \\
& X_{j, j j_{, x_{m}}}\left(d_{11} b_{i} b_{j j j}+d_{33} c_{i} c_{j j j}\right)+ \\
& \left.\left.Y_{j, j j_{, x_{m}}}\left(d_{12} b_{i} c_{j j j}+d_{33} c_{i} b_{j j j}\right)\right) \int_{A} \partial L_{j j j} \partial \tau\right],
\end{aligned}
$$$$
K_{(2 i) j_{, x_{m}}^{* p l b}}=\sum_{j j=1}^{3} \sum_{j j j=1}^{3}\left[\left(c_{i, x_{m}}\left(d_{12} b_{j j j} X_{j, j j}+d_{22} c_{j j j} Y_{j, j j}\right)+\right.\right.
$$$$
c_{j j j_{, x_{m}}}\left(d_{33} b_{i} X_{j, j j}+d_{22} c_{i} Y_{j, j j}\right)+
$$$$
X_{j, j j_{., x_{m}}}\left(d_{12} c_{i} b_{j j j}+d_{33} b_{i} c_{j j j}\right)+
$$$$
\left.\left.Y_{j, j j_{,} x_{m}}\left(d_{22} c_{i} c_{j j j}+d_{33} b_{i} b_{j j j}\right)\right) \int_{A}^{\partial N_{j j z}} \partial \tau, d \tau\right],
$$$$
i=1,2 ; \quad j=1,2, \ldots, 9 \text {. }
$$

Derivatives of loading.

"Lumped" distribution of uniformly distributed and perpendicular to the element plane loading to the element nodes was used at present:

$$
\mathrm{P}_{i}=q\left\{\begin{array}{llllll}
0 & 0 & A / 3 & 0 & 0 & 0
\end{array}\right\}^{\mathrm{T}},
$$

$i=1,2,3$.

Hence the derivatives of nodal loading are:

$$
\mathrm{P}_{i . t_{m}}=\frac{9}{3} A_{, t_{m}} .
$$

Derivatives of deformation energy density.

Those derivatives at arbitrary points of finite element mesh are determined by stress level at the same points:

$$
\begin{aligned}
& u^{e}{ }_{, t_{m}}=\sigma_{1, I_{m}}\left(\sigma_{1} c_{11}^{p l}+\sigma_{2} c_{21}^{p l}\right)+ \\
& \sigma_{2, t_{m}}\left(\sigma_{1} c_{12}^{p l}+\sigma_{2} c_{22}^{p l}\right)+\sigma_{3, l_{m}} \sigma_{3} c_{33}^{p l}+ \\
& \sigma_{4, t_{m}}\left(\sigma_{4} c_{11}^{b}+\sigma_{5} c_{21}^{b}\right)+ \\
& \sigma_{5, t_{m}}\left(\sigma_{4} c_{12}^{b}+\sigma_{5} c_{22}^{b}\right)+\sigma_{6, t_{m}} \sigma_{6} c_{33}^{b},
\end{aligned}
$$

where $c_{i j}$ are for the elements of inverse elasticity matrix.

The stress derivatives, needed for (12), can be expressed through the element stress matrices $\left[\sigma^{e}\right]$ and the displacements of element nodes $\left\{\delta^{e}\right\}$ :

$$
\boldsymbol{\sigma}^{\mathbf{e}}{ }_{t_{m}}=\left[S^{e}\right]_{t_{m}} \delta+\left[S^{e}\right] \boldsymbol{\delta}_{. t_{m}},
$$

where the derivatives of stress matrix are to be obtained in a manner similar to the used for expressions (8) and (9).

Derivatives for material and cross-section optimization.

Sensitivity analysis for this kind of problems requires: derivatives of all aforementioned characteristic quantities with regard to the orthotropy angles for the problem of orthotropy axes orientation; derivatives with regard to the laminae surface coordinates $z$ - for pliesstacking sequence problem, and derivatives with regard to the thickness coefficients - for the problem of laminae thickness optimization. All those derivatives render relatively simply expressions, because they are governed by the elements. of elasticity matrices that are the only terms depending on design parameters.

Now all necessary derivatives for the general sensitivity equation (5) are ready.

\subsection{Optimization technique}

The "move limit" technique [6] is chosen for the relation of design parameters to the mathematical programming methods. Two limit sets (absolute and oneiteration) on all design variables status are imposed. The first set of constraint limits the final shape of problem, while the second allows for linearization of problems in one iteration. 


\subsection{Formulations of problems}

Shape optimization problem.

The coordinates of design nodes were chosen for the design parameters of the problem. Only the nodes on the plate contour comprise the design nodes vector, on which positions of all remaining mesh points depend. The aim of optimization is to minimize maximum deformation energy density $u_{\text {max }}$ by gradually changing shape of the plate under the constraints:

1) deformation energy density everywhere in the structure does not exceed $u_{\max }$;

2) design changes do not exceed absolute and oneiteration move limits;

3) area of model is constant.

Problem of orthotropy angles orientation.

Design parameters for the problem are angles (in global coordinate system) of orthotropy axes of plate laminae. Formulation of problem is identical to previous model except for the third constraint.

Problem of cross-section layout optimization.

The optimal positions of certain layer (layers) are sought in the overall plies-stacking sequence of layers. Here the laminae coordinates $z$ play the role of design parameters. This MIN-MAX problem is formulated in the same way as the shape optimization problem apart from distinct third constraint:

$$
h_{i}+\ldots+h_{N o p t}=\text { const }
$$

when

$$
\begin{gathered}
1 \leq i \leq N_{o p t}, \\
i \leq N_{o p t} \leq N_{l},
\end{gathered}
$$

with $h$ for thicknesses of laminae to be optimized, $N_{o p}$ for the number of optimized layers, and $N_{1}$ for overall number of layers.

\section{Problem of laminae thicknesses optimization.}

The thickness coefficients of separate finite element comprise the design parameters for the problem; ie, plate of step-wise thickness is modelled in optimization process. This kind of formulation, evidently, can not yield practical results, however it will be used later on in the topology optimization. The problem is restraint by the same first two constraints and the third constraint on the constant plate volume.

Merit function and constraints are put in the matrix form required by Simplex procedure [19]. Evidently, all problems are non-linear and are solved iteratively.

\subsection{Algorithm for shape and cross-section layout optimization}

1) Begin.

2) Pre-processing (input, finite element meshing, move limits setup).

3) Optimization Ioop:
DO WHILE optimum criterion does not converge:

3.1) Statics (formulation of structural matrices, imposing boundary conditions, solution of equations for nodal displacements, stresses and energy density).

3.2) Sensitivity analysis loop:

DO $\mathrm{i}=1$, number of design parameters

(Sensitivity analysis; obtaining derivatives of nodal displacements and energy density with regard to the design parameters).

3.3) END DO of sensitivity analysis loop.

3.4) SIMPLEX procedure (formation of matrices, solution, deciphering results).

3.5) Post-processing (appropriate changes of shape/ cross-section layout, regeneration of finite element mesh).

4) END DO of optimization loop.

5) Post-processing of results.

6) END.

\section{Topology optimization of laminated bending plate}

The saltatory changes of plate topology distinguish those problems from the shape optimization. The twolevel iterative algorithm is suggested: in each iteration, at first, the plate thickness optimization is performed according to algorithm 4.5., at second, the diminishing finite elements are eliminated. If there are several finite elements of the same diminishing thickness, the element exposing lowest deformation energy density should be eliminated. In the topology optimization the design area has to be defined in each iteration due to computational reasons.

\subsection{Algorithm for topology optimization}

1) Begin.

2) Pre-processing (input, finite element meshing).

3) Topology optimization loop

DO WHILE maximum energy density in the iteration does not converge:

3.1) Loop for optimization of elements thicknesses: DO $\mathrm{i}=1$, given number of iterations

3.1.1) Statics

3.1.2) Calculation of stresses and energy densities.

3.1.3) Sensitivity analysis loop: DO $\mathrm{i}=1$, number of design parameters (Sensitivity analysis; obtaining derivatives with regard to the design parameters)

3.1.4) END DO of sensitivity analysis loop.

3.1.5) SIMPLEX procedure.

3.1.6) Deciphering results. Alterations of design parameters

3.2) END DO of thicknesses optimization loop.

3.3) Distinguishing elements of minimum thickness $H_{i}=k^{e} H_{\min }$ : 
3.4) Check for influence of element to be eliminated on maximum energy density:

3.4.1) Eliminating element.

3.4.2) Regenerating of finite element mesh.

3.4.3) Calculation of stresses and energy densities.

3.4.4) IF (after elimination of element the energy density does not increase)

THEN eliminate element, equalize thickness of all plate compensating volume of eliminated element.

ELSE regenerate data status before element elimination, increase of coefficient $k^{e}$, GO TO 3.1.

END IF.

4) END DO of topology optimization loop.

5) Post-processing of results.

6) END.

\section{Numerical examples}

Here we provide numerical examples illustrating all four aforementioned categories of optimization problems. The dimensionless units are used in all solutions.

\subsection{Examples of shape optimization}

Test 1. Optimization of cantilever plate under inplane loading.

Laminated plate (Fig 2) made of three orthotropic graphite/epoxy layers is optimized. For the sake of transparency of expected results, all layers at initial stage are oriented by the same orthotropy angles. The move limits for alterations of design parameters in one iteration are adjusted to the extent of non-linearity so that the predictions of Simplex procedure on the future behaviour of structure do not differ remarkably from finite element solution.

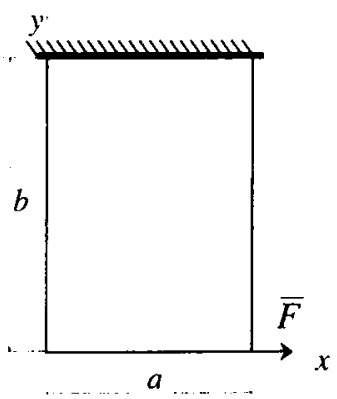

Fig 2. Computational scheme

\section{Data:}

$a=b=10, F_{x}=100$, number of layers 3 , laminae materials (1)/(1)/(1);

material properties:

1) $\mathrm{E}_{11}=0,181 \mathrm{E}+12, \mathrm{E}_{22}=0,103 \mathrm{E}+11, \mathrm{G}_{12}=0,717 \mathrm{E}+10$, $v_{12}=0,28$

thicknesses of layers: $0,05 / 0,01 / 0,01$;

angles of orthotropy axes: $90^{\circ} / 90^{\circ} / 90^{\circ}$; all coordinates of nodes on the contour of plate (except for the node under concentrated load) are chosen to be the design parameters.
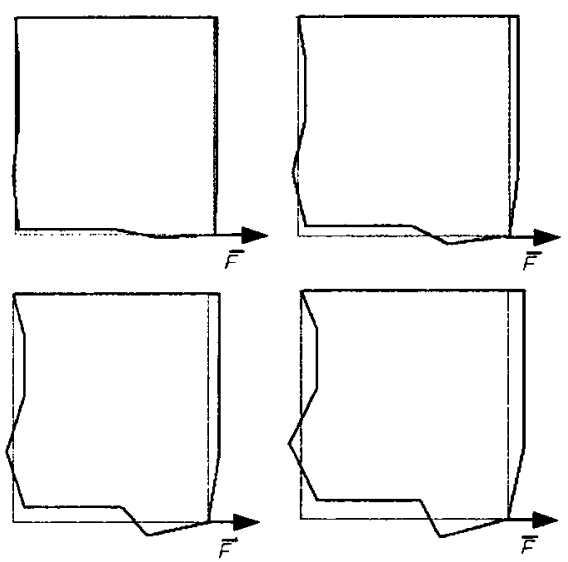

Fig 3. History of shape optimization (iterations 500,1000 , $1500,2000)$, test 1

$$
\begin{aligned}
& u_{\max }^{1 \text { iter }}=0,159259 \cdot 10^{-5}, \\
& u_{\max }^{2209 \text { iter }}=0,708378 \cdot 10^{-6} .
\end{aligned}
$$

The maximum deformation energy decreases by $55 \%$ in optimization process (Fig 3,4). The solution is stopped when the design parameters reach the absolute move limits.

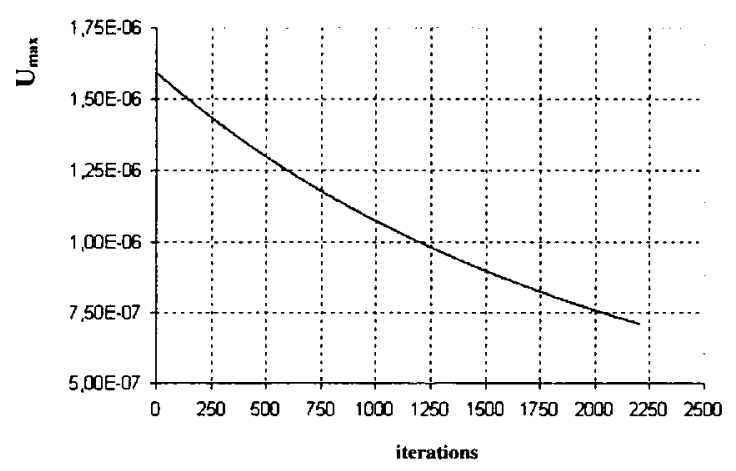

Fig 4. History of energy density alteration, test 1

Test 2. Optimization of cantilever plate under outof-plane loading.

The standard test for this kind of optimization problems is a quadratic plate with a hole in the centre; the shape of the hole to be optimized (Fig 5). Again, the plies-stacking sequence and material properties are the same as in test 1 . Angles of orthotropy axes are $0 \% \%$ $0^{\circ}$. Dimensions of plate are: side length $l=20$, radius of hole $r=5$.

The plate is clamped along contour and loaded by uniformly distributed loading. Design parameters are the coordinates of all nodes on the contour of hole. Absolute move limits on status of design nodes are chosen so that the final shape of hole does not distort remarkably the initial finite element mesh. In case of those moderate 
limits the regeneration of finite element mesh in the optimization process can be avoided. Only one quarter of the plate is modelled applying appropriate symmetry conditions to the sections.

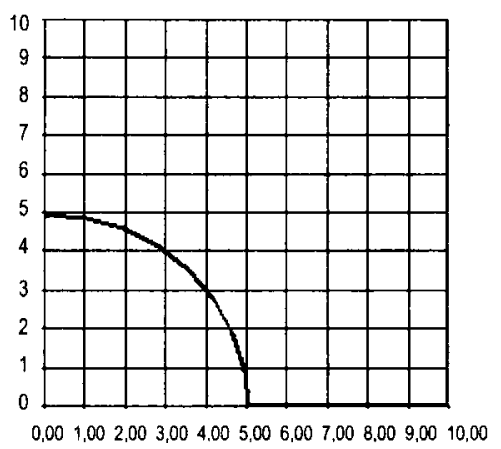

Fig 5. Computational scheme for shape optimization under bending loading, test 2

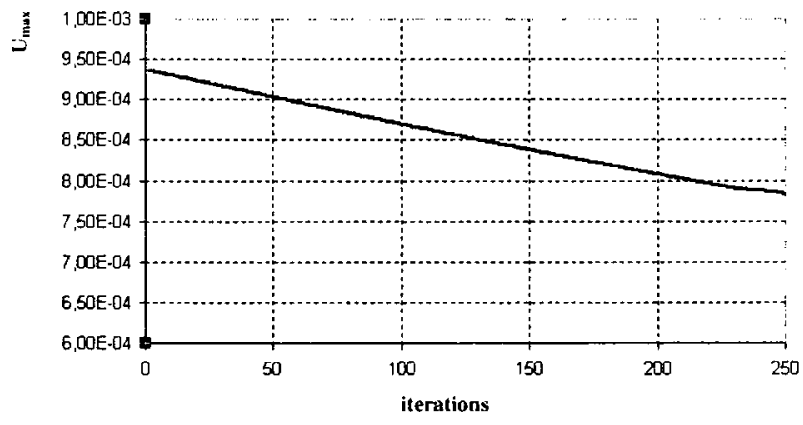

Fig 6. History of energy density alteration, test 2

$$
\begin{aligned}
& u_{\max }^{\text {iter }}=0,93637 \cdot 10^{-5}, \\
& u_{\max }^{250 \text { iter }}=0,78281 \cdot 10^{-5} .
\end{aligned}
$$

The maximum deformation energy density decreases by $16,4 \%$ in optimization process (Fig 6,7).
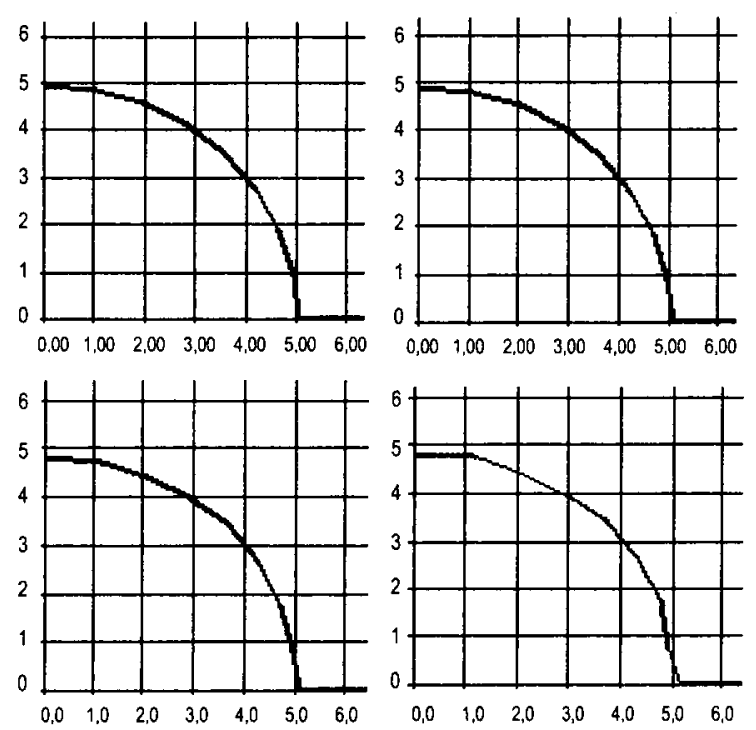

Fig 7. Alteration of hole shape (iterations 100, 150, 200 250 ), test 2

\subsection{Examples of material optimization}

Test 3. Optimization of orthotropy angles in layers of cantilever plate under in-plane loading.

The cantilever plate made of four different layers (glass exopy/hypothetical material) is optimized. All materials expose indistinct orthotropy properties. Optimization of orientation angles of orthotropy axes is carried out in two stages: first, quasioptimal solution is found by the trial variations of orthotropy angles $-45^{\circ}-0^{\circ}-45^{\circ}$ $-90^{\circ}$; second, sensitivity analysis is executed. This scheme assures the avoidance of local solutions.

Note, for this particular test the angles $0^{\circ} / 0^{\circ} / 0^{\circ} / 0^{\circ}$ are obtained. This result is in good agreement with "engineering suspicion": the orthotropy axis corresponding to the better material properties is oriented lengthwise the concentrated load direction.

\section{Data:}

$a=b=10, F_{x}=100$, number of layers 4 , laminae materials $(1) /(2) /(2) /(1)$;

material properties:

1) $\mathrm{E}_{11}=0,538 \mathrm{E}+11, \mathrm{E}_{22}=0,179 \mathrm{E}+11, \mathrm{G}_{12}=0,896 \mathrm{E}+10$, $v_{12}=0,25$;

2) $\mathrm{E}_{11}=0,538 \mathrm{E}+10, \mathrm{E}_{22}=0,179 \mathrm{E}+10, \mathrm{G}_{12}=0,896 \mathrm{E}+09$, $v_{12}=0,25$;

thicknesses of layers: $0,01 / 0,02 / 0,03 / 0,04$;

angles of orthotropy axes: $0^{\circ} / 0^{\circ} / 0^{\circ} / 0^{\circ}$;

design parameters: orientation angles of orthotropy angles of all layers.

$$
\begin{aligned}
& u_{\max }^{1 \text { iter }}=0,20778 \cdot 10^{-5}, \\
& u_{\max }^{297 \text { iter }}=0,19758 \cdot 10^{-5} .
\end{aligned}
$$

The final orthotropy angles are as follows:

$-7,214 \%-14,178 \%-14,178 \%-2,126^{\circ}$.

The maximum deformation energy density decreases by $4,91 \%$ (Fig 8 ) in comparison with quasioptimal solution $0 \% 0^{\circ} / 0^{\circ} / 0^{\circ}$.

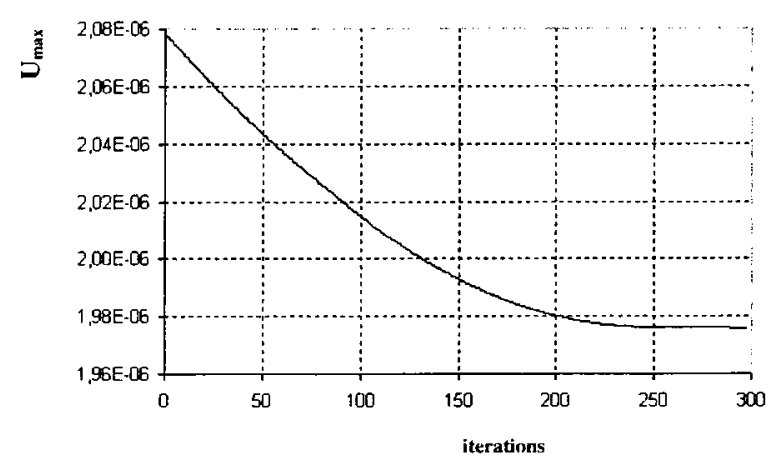

Fig 8. History of energy density alteration, test 3

Test 4. Optimization of plies-stacking sequence of cantilever plate under out-of-plane loading.

Cantilever plate (Fig 2) made of five different layers is optimized. The materials are the same as in test 3 . 
Design parameters are the 2 vertical coordinates of the internal surfaces of layers. The absolute move limits are determined by vertical coordinates of external cross-section surfaces. In this particular test the condition on constant summary thickness of layers 2 and 4 are led in. In essence, the optimal position of those layers in the crosssection is sought.

\section{Data:}

$a=b=10, \quad F_{x}=100$, number of layers 5 , laminae materials (2)/(1)/(2)/(1)/(2);

material properties: see test 3 ;

thicknesses of layers: $0,005 / 0,01 / 0,1 / 0,02 / 0,015$;

angles of orthotropy axes $0^{\circ} / 0^{\circ} / 0^{\circ} / 0^{\circ}$.

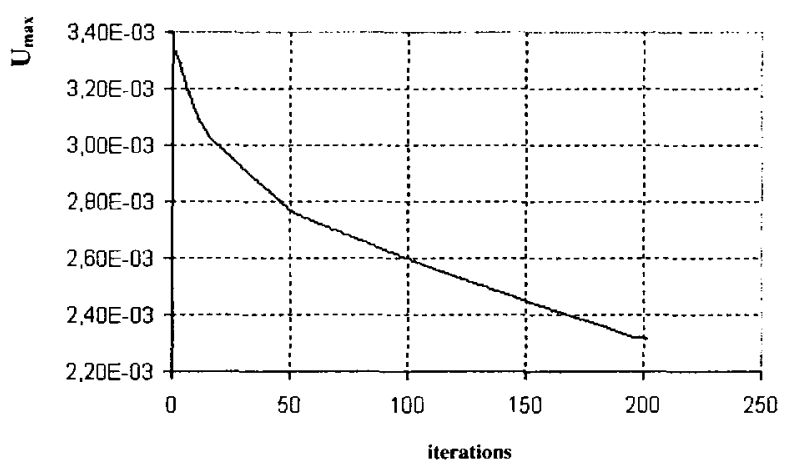

Fig 9. History of energy density alteration, test 4

$$
\begin{aligned}
& u_{\max }^{1 \text { iter }}=0,33322 \cdot 10^{-2}, \\
& u_{\max }^{202 \text { iter }}=0,23181 \cdot 10^{-2}
\end{aligned}
$$

After 200 iterations the cross-sectional nomenclature becomes:

0,0/0,015/0,12/0,015/0,0.

The maximum deformation energy density decreases by $30,4 \%$ (Fig 9 ).

\subsection{Examples of topology optimization}

Huge computer resources are required for this kind of optimization of laminated plates, therefore in this paper we restrict ourselves with rather coarse finite element meshes. However, the tests are sufficient to illustrate the principal ideas and actions of optimization algorithms.

Test 5. Topology optimization of laminated cantilever plate under in-plane loading.

Cantilever plate (Fig 2) made of three different layers is optimized. The outer layers are made of graphite/ epoxy, while the middle layer is isotropic.

In the first step of topology optimization the constraint on the design area is imposed: only the inner area is to be optimized. Then in the second step the design area involves whole plate. Seven thickness iterations are performed in each topology iteration.
Data:

$a=b=8, F_{y}=100$, number of layers 3 , laminae materials (1)/(2)/(1);

material properties:

1) $\mathrm{E}_{11}=0,207 \mathrm{E}+12, \mathrm{E}_{22}=0,517 \mathrm{E}+10, \mathrm{G}_{12}=0,259 \mathrm{E}+10$, $v_{12}=0,25$

2) $\mathrm{E}_{11}=0,100 \mathrm{E}+10, \mathrm{E}_{22}=0,100 \mathrm{E}+10, \mathrm{G}_{12}=0,385 \mathrm{E}+10$, $v_{12}=0,3$;

thicknesses of layers: $0,01 / 0,1 / 0,01$;

angles of orthotropy axes: $90^{\circ} / 0^{\circ} / 90^{\circ}$,

The result of topology alteration is presented in Fig 10, where by white colour the eliminated elements are indicated, while by black colour the elements to be retained.

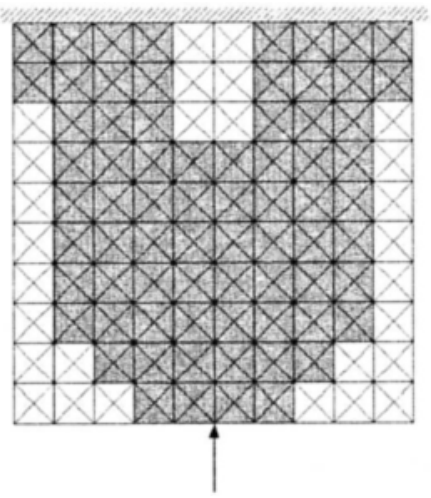

Fig 10. Final topology of plate, test 7

Results of optimization are:

$$
\begin{aligned}
& u_{\max }^{1 \text { iter }}=0,91425 \cdot 10^{-5}, \\
& u_{\max }^{14 \text { iter }}=0,68804 \cdot 10^{-5} .
\end{aligned}
$$

Maximum deformation energy density decreases by $24,7 \%$ in overall 70 iterations (14 topology alterations among them).

Testas 6. Optimization of laminated slab under inplane loading.

This is well-known Michell problem [20], for which the optimal solution is a discrete structure as shown in Fig 11.

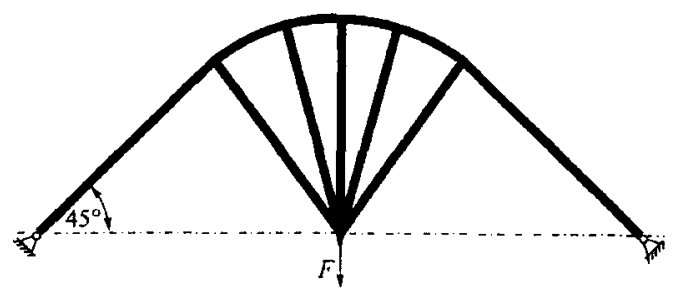

Fig 11. Optimal topology of Michell structure made of isotropic material

Here the similar problem will be solved for the structure made of orthotropic laminated material with better properties in longitudinal direction. 
Data:

$L_{x}=2, \quad L_{y}=0,5 ; F_{y}=100$, number of layers 3 ; laminae materials $(\mathbf{1}) /(2) /(1)$;

material properties:

1) $\mathrm{E}_{11}=0,207 \mathrm{E}+12, \mathrm{E}_{22}=0,517 \mathrm{E}+10, \mathrm{G}_{12}=0,259 \mathrm{E}+10$, $v_{12}=0,25$

2) $\mathrm{E}_{11}=0,100 \mathrm{E}+10, \mathrm{E}_{22}=0,100 \mathrm{E}+10, \mathrm{G}_{12}=0,385 \mathrm{E}+10$, $v_{12}=0,3$;

thicknesses of layers: $0,05 / 0,1 / 0,05$;

angles of orthotropy axes: $0^{\circ} / 0^{\circ} / 0^{\circ}$;

design area: all structure except for supported nodes.

The resulting topology is similar to the Michell structure and is shown in Fig 12. As expected, the slab's height decreases due to prevailing longitudinal material properties.

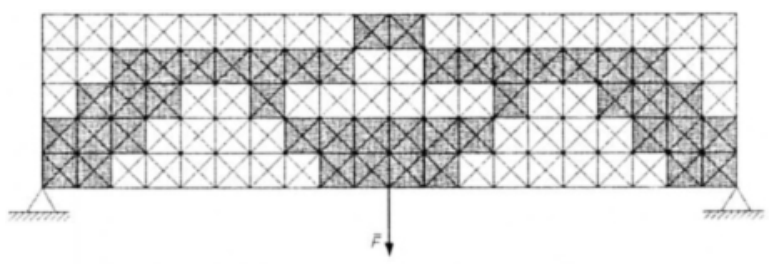

Fig 12. Final topology of slab (after 28 topology iterations), test 6

$$
\begin{aligned}
& u_{\max }^{1 \text { iter }}=0,4618 \cdot 10^{-3}, \\
& u_{\max }^{28 \text { iter }}=0,1976 \cdot 10^{-3} .
\end{aligned}
$$

The maximum energy density decreases by $57,2 \%$ in optimization process.

Test 7. Optimization of quadratic laminated plate under out-of-plate concentrated load at the centre.

The plate is clamped along the whole contour. The material of structure is non-symmetrical composite (Fig 13). The central node of structure (at which the load is applied) is fixed in the optimization process; the design area is all remaining surface of plate.

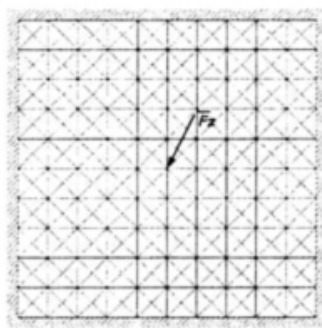

Fig 13. Computational scheme, test 7

Data:

$L_{x}=8, L_{y}=8 ; F_{z}=100$, numbers of layers 3 ; materials (i) $/(2) /(1)$;

materials properties:

1) $\mathrm{E}_{11}=0,207 \mathrm{E}+12, \mathrm{E}_{22}=0,517 \mathrm{E}+10, \mathrm{G}_{12}=0,259 \mathrm{E}+10$, $v_{12}=0,25$;

2) $\mathrm{E}_{11}=0,100 \mathrm{E}+10, \mathrm{E}_{22}=0,100 \mathrm{E}+10, \mathrm{G}_{12}=0,385 \mathrm{E}+10$, $v_{12}=0,3$; thicknesses of layers: $0,025 / 0,1 / 0,05$; angles of orthotropy axes: $0^{\circ} / 0^{\circ} / 90^{\circ}$

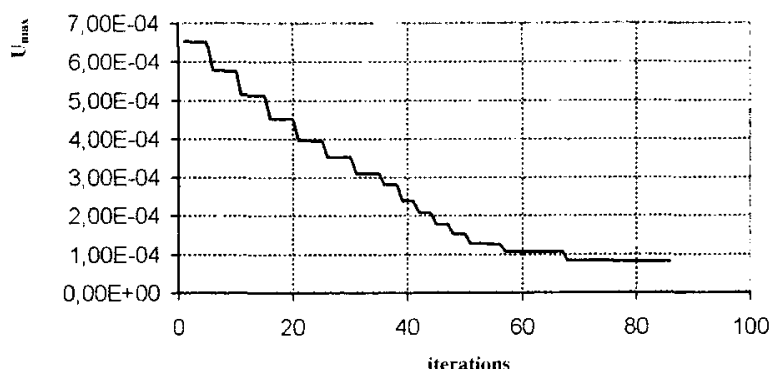

Fig 14. History of energy density alteration, test 7

Results of solution are provided by Fig 14-17.

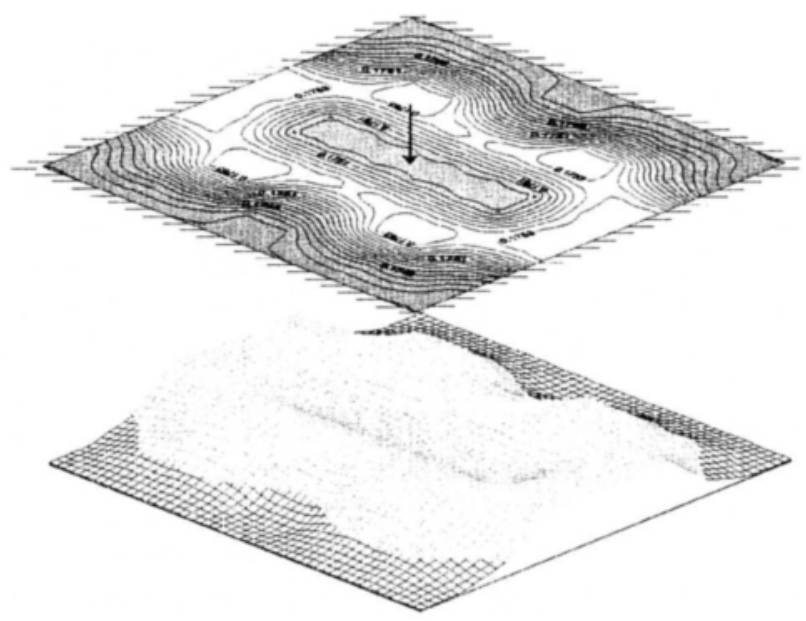

Fig 15. Optimized surface of plate before the 1st topology alteration, test 7

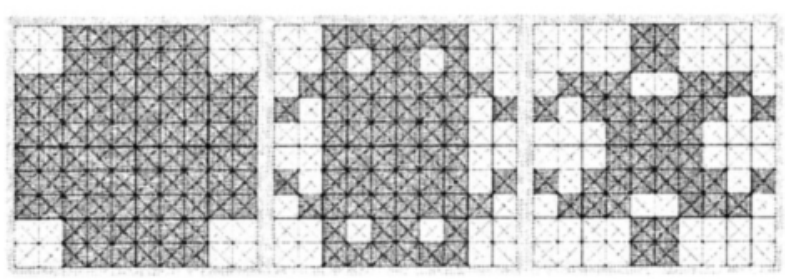

Fig 16. History of topology optimization $(5,10,15$ topology iterations), test 7

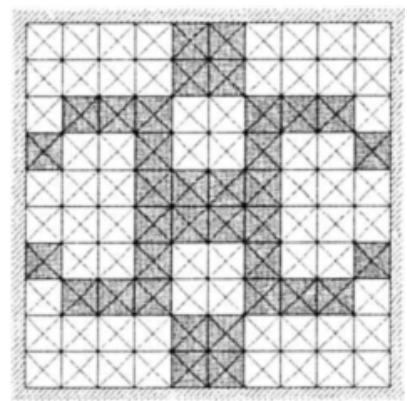

Fig 17. Final topology of plate, test 7 


$$
\begin{aligned}
& u_{\max }^{1 \text { iter }}=0,65287 \cdot 10^{-3}, \\
& u_{\max }^{17 \text { iter }}=0,05321 \cdot 10^{-3} .
\end{aligned}
$$

Thicknesses of laminae after optimization: $0,0695 /$ $0,486 / 0,139$.

Maximum energy density decreases by $91,8 \%$ in optimization process.

As seen from Fig 14, after each topology alteration a distinct decrease of energy density is obtained. The final topology of plate is in good agreement with engineering expectations. The algorithm has been verified by solution of anti-symmetric problem (orthotropy angles $90^{\circ} / 90^{\circ} / 0^{\circ}$ ). As expected, solution yields exactly antisymmetric topology.

\section{Conclusions}

The all different feasible optimization problems of thin orthotropic laminated bending plate are formulated and solved in a unified manner: shape optimization, material optimization (ie, optimization of orthotropy angles is laminae, optimization of laminae stacking sequence in cross-section), sizing (plate thickness) optimization, and topology optimization. The plate is modelled by combined finite element comprising layered Discrete Kirchhoff theory element (DKT) as a bending part, and Constant Strain Triangle (CST) as a membrane part. The uniform distribution of deformation energy density is chosen as an optimum criterion for all problems. Semianalytical sensitivity analysis is performed for all design parameters: closed-form derivatives are obtained for stiffness, strain, stress, energy, and loading matrices. Original algorithms are suggested for optimization, where the thickness optimization plays the role of geometrical filter on the intermediate results of topology optimization.

The presented optimization results illustrate the accuracy and workability of formulated element and algorithms. The suggested simple finite element and its numerically effective formulation is especially attractive for topology optimization, where the required computer resources limit the size and complexity of problems.

\section{References}

1. Rozvany G. I. N. Aims, scope, methods, history and unified terminology of computer-aided topology optimization in structural mechanics. Struct. Multidisc. Optimization, Vol 21, 2001, Springer-Verlag, p 90-108.

2. Rozvany G. I. N., Olhoff N., Cheng K.-T., Taylor J. E. On the Solid Plate Paradox in Structural Optimization. Structural Optimization, Vol 10, 1982, p 1-32.

3. Xie Y. M., Steven G. P. A simple evolutionary procedure for structural optimization. Comput.\& Struct., 1993, p 885896.
4. Sigmund O. Topology optimization: a tool for the tailoring of structures and materials. Phil. Trans. Roval Society, Vol 358, London, 2000, p 1031-1048.

5. Francavilla A., Ramakrishnan C.V., Zienkiewicz O.C. Optimization of shape to minimise stress concentration. $J$. of Strain Analysis, Vol 10, 1975, p. 63-70.

6. Pedersen P. Design for Minimum Stress Concentration Some Practical Aspects. Structural Optimization. Kluwer Academic, 1988, p 225-232.

7. Pedersen P. Concurrent Engineering Design of and with Advanced Materials. DCAMM Report. The Technical University of Denmark, 1991. 56 p.

8. Belevičius R. Shape Optimization of Laminated Orthotropic Plate Structures. Mech. of Composite Mater., Vol 29, 1993. p 537-546.

9. Liang Q. Q., Xie Y. M., Steven G. P. A performance index for topology and shape optimization of plate bending problems with displacement constraints. Struct. Multidisc. Optimization, Vol 21, 2001, Springer-Verlag, p 393-399.

10. Querin O. M., Xie Y. M., Steven G. P. Evolutionary Structural Optimisation using a Bidirectional Algorithm. Engineering Computations, Vol 15, 1998, p 1031-1048.

11. Liu J. S., Parks G. T., Clarkson P. J. Metamorphic development: a new topology optimization method for continuum structures. Struct. Multidisc. Optimization, Vol 20, 2000, Springer-Verlag, p 288-300.

12. Zhou M., Rozvany G. I. N. On the validity of ESO type methods in topology optimization. Struct. Multidisc. Optimization, Vol 21, Springer-Verlag, 2001, p 80-83.

13. Bendsoe M. P. Optimization of structural topology, shape and material. Berlin: Springer, 1995. 271 p.

14. Bendsoe M. P., Kikuchi N. Generating optimal topologies in optimal design using a homogenization method. Computational Methods in Applied Mechanics and Engineering, Vol 71, 1988, p 197-224.

15. Bendsoe M. P., Sigmund O. Material interpolation schemes in topology optimization. Archive of Applied Mechanics, Vol 69, 1999, Springer-Verlag, p 635-654.

16. Goldberg D. E. Genetic algorithms in search, optimization and machine learning. Addison-Wesley Pub Co, 1989. $412 \mathrm{p}$.

17. Autio M. Determining the real lay-up of a laminate corresponding to optimal lamination parameters by genetic search. Struct. Multidisc. Optimization, Vol 20, SpringerVerlag, 2000, p 301-310.

18. Belevičius R., Michnevič E., Rusakevičius D. Layered Orthotropic Plate Bending Element of Discrete Kirchhof Theory. In: XXXVII Sympozium "Modeling in Mechanics", Gliwice, Poland, 1998, p 29-37.

19. Belevičius R. Computer Algebra in Finite Element Method. Vilnius: Technika, 1994. 184 p.

20. Michell A. G. M. The limit economy of material in frame structures. Phil. Mag., Vol 8, 1904, p 589-597. 